\title{
Anorexia nervosa and nutritional assessment: contribution of body composition measurements
}

\author{
Lama Mattar ${ }^{1,2}$, Nathalie Godart ${ }^{3}$, Jean Claude Melchior ${ }^{4,5}$ and Claude Pichard ${ }^{6 *}$ \\ ${ }^{1}$ INSERM U669, Maison de Solenn, 97 Boulevard De Port Royal, 75014 Paris, France \\ ${ }^{2}$ Université Paris-Sud and Université Paris Descartes, UMR-SO669, 75679 Paris, France \\ ${ }^{3}$ Service de psychiatrie, Institut Mutualiste Montsouris, 42 Boulevard Jourdan, 75014 Paris, France \\ ${ }^{4}$ Unité de Nutrition clinique-Maladies infectieuses, Département de Médecine aiguë, Hôpital Raymond Poincaré, 92380 \\ Garches, France \\ ${ }^{5}$ Université Versailles St Quentin en Yvelines, 94415 Villejuif, France \\ ${ }^{6}$ Unité de Nutrition, Hôpitaux Universitaires de Genève, Rue Gabrielle-Perret-Gentil, 4, 1211 Geneva 14 , Switzerland
}

\section{Abstract}

The psychiatric condition of patients suffering from anorexia nervosa (AN) is affected by their nutritional status. An optimal assessment of the nutritional status of patients is fundamental in understanding the relationship between malnutrition and the psychological symptoms. The present review evaluates some of the available methods for measuring body composition in patients with AN. We searched literature in Medline using several key terms relevant to the present review in order to identify papers. Only articles in English or French were reviewed. A brief description is provided for each body composition technique, with its applicability in AN as well as its limitation. All methods of measuring body composition are not yet validated and/or feasible in patients with AN. The present review article proposes a practical approach for selecting the most appropriate methods depending on the setting, (i.e. clinical $v$. research) and the goal of the assessment (initial $v$. follow-up) in order to have a more personalised treatment for patients suffering from AN.

Key words: Anorexia nervosa: Body composition: Nutritional assessment: Bioelectrical impedance analysis: Dual-energy X-ray absorptiometry: BMI

Anorexia nervosa (AN) is one of the most common chronic diseases in adolescents ${ }^{(1)}$ and can also persist into adulthood. AN is associated with severe somatic symptoms due to restrictive eating and abnormal behaviour such as purging, potomania, over-exercising, laxative abuse, etc $^{(2)}$. Malnutrition is a serious somatic aspect of AN; its amelioration is a fundamental part of the treatment: clinically, malnutrition affects the psychiatric condition of the patient ${ }^{(3,4)}$. Similarly, in research, questions have been raised on the relationship between malnutrition and the psychological symptoms in AN (anxiety, depression and obsessionality). However, in previous studies, the methodology was questionable, as only body weight had been used for the nutritional assessment ${ }^{(3-6)}$. Complementary to body weight measurements, assessing the body's muscle and fat stores is fundamental for the diagnosis and treatment of malnutrition in AN as well as for assessing the patient's response to nutritional and other therapies.
Generally, the two-compartment (2-C) model is used in studies that assess body composition in AN. The 2-C model separates the composition of the body into fat mass (FM) and fat-free mass (FFM) which requires the assumptions that body hydration level and bone mineral content are both stable. As those assumptions cannot constantly be done in AN patients, the 2-C model can thus lead to potentially large errors in estimates of components of body composition ${ }^{(7,8)}$.

Recently, the four-compartment (4-C) model for assessing body composition has allowed researchers to measure variations in hydration levels and bone mineral contents ${ }^{(9)}$. By adding measurements only from direct and scientifically accepted methods of total body water (for example, via ${ }^{2} \mathrm{H}$ dilution), bone mineral density (for example, via dualenergy X-ray absorptiometry (DXA)), and body density (for example, via underwater weighing (UWW)), it is possible to measure individual variations in minerals and

\footnotetext{
Abbreviations: AN, anorexia nervosa; BIA, bioelectrical impedance analysis; 4-C, four-compartment; CT, computed tomography; DXA, dual-energy X-ray absorptiometry; FFM, fat-free mass; FM, fat mass; MUAC, mid-upper arm circumference; SFT, skinfolds thickness; UWW, underwater weighing; WHR, waist-to-hip ratio.
} 
water $^{(10)}$. In theory, this should lead to a more accurate measurement of percentage body fat in AN. However, to our knowledge, and because of practical constraints, no study has been done in AN using the 4-C model although it has been done in populations such as in elderly patients ${ }^{(11)}$.

The present review will describe the impact of AN on body composition and will appraise the accuracy and applicability of the different methods for measuring body composition such as anthropometric measurements (for example, body weight, skinfolds measurements), bioelectrical impedance analysis (BIA), DXA and some other methods for research use only. In the final section, recommendations are made on which method to use depending on the setting, (i.e. clinical $v$. research) and the goal of the assessment (initial $v$. follow-up).

\section{Body composition alterations in anorexia nervosa}

AN has a deep impact on body composition ${ }^{(12)}$. Adaptive changes to low body weight have an impact on bone metabolism and decrease bone mass density ${ }^{(13)}$. Mazess et $a l .{ }^{(14)}$ found that absolute bone mineral content was $25 \%$ lower in AN patients when measured using dualphoton absorptiometry. The decreased bone mineral density in AN is related to the loss of muscle mass and reduced muscle strength rather than the bone mineral density itself $^{(15,16)}$. The FM, FFM and total body water are not all affected to the same extent due to the variable impact of factors such as physical activity, vomiting, laxative abuse and diet. For instance, patients with AN who achieve an extreme weight loss by semi-starvation can have a different body composition profile from patients who achieved the weight loss primarily through excessive exercise ${ }^{(17)}$. Based on The American Council on Exercise, normal fat percentages for adult women are between 21 and $31 \%$ and for men between 14 and $25 \%$ of total body weight, depending on age and exercise. In patients with AN, alterations are represented mainly by an extensive loss of FM, suggesting a preferential loss of body fat ${ }^{(14,18)}$. Nevertheless, there is also a loss in FFM but the extent of the loss depends on the level of exercise maintained by the patient ${ }^{(19)}$. Concerning weight gain, Iketani et al. ${ }^{(20)}$ assessed by dual-photon absorptiometry twenty-one AN patients and showed that after weight gain and resumption of menses, most of the weight gain consisted of FM (80.6\% of total weight gained) compared with lean body mass (19.3\% of total weight gained). Also, Probst et al. ${ }^{(21)}$ who assessed AN patients by UWW and skinfolds thickness (SFT) found that after 23 weeks of treatment, patients gained more in FM $(55.5 \%$ of total weight gained) than in FFM (44.5\% of total weight gained). Besides, Russell et $a l^{(22)}$ found that the regained lean body mass assessed by SFT and anthropometric measurements had a complete restored function. On the contrary, an older study, in which body composition was assessed by SFT and by neutron activation analysis, found that FM accounts less than lean body mass to weight gain ${ }^{(23)}$.

As a result, it is crucial to obtain the parameters of body composition in relative percentages of the total body mass in order to monitor accurately the response of the fat and lean components to therapy ${ }^{(24,25)}$.

\section{Body composition-measuring techniques in anorexia nervosa}

Anthropometric techniques, BIA and DXA are clinically available methods for measuring body composition and will be discussed below. Many of those methods have limitations in AN. Practitioners can use some of these techniques whereas others are limited to research settings.

\section{Basic anthropometric measurements: body height, weight and BMI}

Brief description of body weight, height and BMI. When measuring body height, the patient should be standing with heels together, arms to the side, legs straight, shoulders relaxed and head in the horizontal plane while the patient is 'looking straight ahead'. Measurement should be rounded to the nearest $0 \cdot 1 \mathrm{~cm}^{(26)}$. Weight is measured in light clothing and the subject should be standing still in the middle of the scale's platform and not touching surrounding objects ${ }^{(27)}$. Measurement reading should be rounded to the nearest of $100 \mathrm{~g}$. Because of diurnal variations (difference reaching up to $2 \mathrm{~kg}$ in adults), a good practice would be to record the time when weight was measured ${ }^{(26)}$. Alternatively, it is easy to determine the body weight in the morning after waking up and having voided the bladder. From the measured height and weight, BMI can be calculated. In children and adolescents, BMI should be used with caution as it is relative to the country's reference growth chart (i.e. Centers for Disease Control growth charts in the USA ${ }^{(28)}$ ) and to the age; a BMI of $17.5 \mathrm{~kg} / \mathrm{m}^{2}$ would be on the $3 \mathrm{rd}$ percentile for a 19-year-old but on the 50th percentile for an 11-year-old ${ }^{(29)}$.

Applicability and limitation of body weight and $B M I$. In cases of severe malnutrition, body weight alone is not a sensitive tool to determine nutritional status ${ }^{(30,31)}$. The loss of body cell mass can be compensated by extracellular fluid accumulation and thus cannot be accurately sensed by body weight measurement ${ }^{(30,32)}$. Trocki \& Shepherd ${ }^{(33)}$ showed that in adolescents with AN, changes in BMI do not correlate well with changes in body composition. Although BMI alone does not differentiate between fat and lean masses in children and teenagers ${ }^{(34)}$, it is correlated with components of body composition. For example, BMI in children correlates more strongly with FM at the upper end of the adiposity range where FM makes up a larger proportion of weight, than at the lower end. Thus in thin children, BMI is a better predictor of lean mass than $\mathrm{FM}^{(35)}$. Cole's index for thinness does not 
quantify malnutrition but rather gives cut-off points under which an individual is classified as thin or undernourished. Nevertheless, body weight for age and height and BMI percentiles have been shown to correlate with the resumption of menses in girls with AN and can therefore be used in setting a target weight. Golden et al. ${ }^{(36)}$ have found that $86 \%$ of patients who achieved a weight goal of $90 \%$ of the average weight for age and height (using the National Center for Health Statistics (NCHS) tables) resumed menses within 6 months. More recently, Golden et al. ${ }^{(29)}$ have set the BMI percentile cut-off points at which adolescents with AN resumed their menses. Of the patients, 50\% resumed their menses when their BMI reached $\geqq$ the 14 th percentiles.

Body weight alone is not a complete assessment tool; however, it is still essential to use it in the initial nutritional assessment and follow-up in clinical settings. Other methods have been developed to measure body composition. Commonly available methods to assess body composition (skinfolds, BIA, DXA) have not yet been validated against a reference $4-\mathrm{C}$ model in $\mathrm{AN}$ patients. This would be important because in patients with $\mathrm{AN}$, body composition extremely alters and they undergo drastic shifts in their electrolytes and hydration status.

\section{Skinfolds thickness, mid-upper arm circumference and waist-to-hip ratio}

Brief description of skinfolds thickness. SFT measurement is an inexpensive and basic tool for determining body composition ${ }^{(37)}$. It consists of measuring the thickness of a double fold of skin and compressed subcutaneous adipose tissue with a caliper at specific points in the body. There are three points that are the most commonly used (triceps, subscapular, supra-iliac); however, there are other possible reference points (i.e. thigh) ${ }^{(27)}$. The measures obtained are then used in prediction equations ${ }^{(27)}$. Because $80 \%$ of the body's adipose tissue is localised under the skin, SFT is a surrogate of body fat percentage ${ }^{(38)}$. From clinical experience, SFT can still be assessed quite well in very lean subjects.

Applicability and limitation of skinfolds thickness in anorexia nervosa. SFT measurements have been frequently used in AN studies ${ }^{(39)}$. Measurements are less affected by the hydration status of the patient compared with other methods, which makes this technique advantageous in AN patients. Disadvantages of SFT are its low sensitivity to short-term nutritional changes ${ }^{(40)}$, to measuring visceral abdominal fat ${ }^{(17)}$ and to detecting changes in regional fat distribution ${ }^{(41)}$. Another general drawback of SFT is the large variability between different observers. As a result, SFT measurement is mainly relevant in healthy populations, especially in an epidemiological research setting ${ }^{(25)}$.

Brief description of mid-upper arm circumference. Another reproducible and easy-to-do method is midupper arm circumference (MUAC). It consists of measuring on the non-dominant arm, midway between the tip of the acromium process and the olecranon using a non-stretch paper measuring tape, the circumference of the arm. It is an indicator of muscle mass ${ }^{(42)}$.

Applicability and limitation of mid-upper arm circumference in anorexia nervosa. Martin et al. ${ }^{(43)}$ have used this method in AN, considering that weight and BMI can misinform on nutritional status, as AN patients can attempt to mislead the physician often by water-loading or discreetly placing weights on their body in order to increase their recorded weight, putting them at risk of complications of malnutrition. They found that MUAC was highly correlated with BMI $(r 0.79 ; P<0.001)$ and individuals with MUAC greater than $20 \mathrm{~cm}$ rarely required hospitalisation.

Brief description of waist-to-hip ratio. Waist-to-hip ratio (WHR) is used as an indicator of central fat distribution. It is the quotient of the waist circumference divided by the hip circumference. One of the accepted reference points to measure the waist circumference is at the level immediately below the lowest ribs and hip circumference is measured below the iliac crest; however, the importance is to always use the same reference point and to standardise the measurements ${ }^{(44)}$.

Applicability and limitation of waist-to-hip ratio in anorexia nervosa. The WHR in AN allows follow-up on regional fat distribution, especially during weight gain $^{(45-47)}$. Forbes et al. $^{(45)}$ were the first to examine body fat distribution in a cross-section of underweight women with AN. Using the WHR as a measure of body fat distribution, they reported no difference in the WHR between women with AN and healthy control subjects. However, Mayer et al. ${ }^{(46)}$ found by measuring the WHR that, immediately after weight restoration to a normal weight, women with AN have an adipose tissue distribution that differs significantly from healthy control subjects.

\section{Bioelectrical impedance analysis}

Brief description of body impedance analysis. The concept of BIA is based on applying to the human body a low-intensity electrical current through electrodes placed on the ankles and wrists. The current passes predominantly through water and electrolytes while it faces resistance through cell membranes and adipose tissue. Resistance and reactance are the main parameters measured. They are used to calculate, using mathematical equations, the FFM. However, it is important to note that those equations are based on the assumption that hydration status is constant: this is not the case in $\mathrm{AN}^{(32,48)}$. The $\mathrm{FM}$ is indirectly measured by subtracting the FFM from the total weight. BIA is an easy-to-perform, reproducible, non-invasive and inexpensive method to measure body composition.

Applicability and limitation of body impedance analysis in anorexia nervosa. BIA is reliable in healthy subjects and ill patients with a stable fluid/electrolytes balance 
(using the appropriate equations). It has not yet been validated for use in extreme cases, such as in severely malnourished (including AN cases) or obese individuals ${ }^{(48,49)}$. Many reference equations have been proposed in different studies, but they were based on either healthy subjects ${ }^{(32)}$ or on a sample poorly representative of the general population. In AN, the variability in extra-cellular and intracellular volume is likely to be responsible for reduced accuracy of BIA measurements and for the unexplained variance of body FFM and leg $\mathrm{FFM}^{(50)}$. Sexual dimorphism is an important factor, which has led to sex specificity in the BIA equations; Sun et al. ${ }^{(51)}$ have studied BIA equations in men and women based on 1801 subjects with BMI between 14 and $39 \mathrm{~kg} / \mathrm{m}^{2}$. They did not restrict their population to very lean subjects. Using Sun's equations, Piccoli et $a l .{ }^{(52)}$ have compared results of body composition measured by SFT and BIA in seventy-four women with a BMI between 10 and $17.5 \mathrm{~kg} / \mathrm{m}^{2}$. They showed that BIA and anthropometric measurements were highly correlated ( $r$ 0.80-0.90; $P<0.05$ ) when BMI values were above $15 \mathrm{~kg} / \mathrm{m}^{2}$. With BMI values less than $15 \mathrm{~kg} / \mathrm{m}^{2}$, there was a disagreement of FM and FFM estimates between the two different methods. They concluded that for patients with AN with a BMI below $15 \mathrm{~kg} / \mathrm{m}^{2}$ down to $10 \mathrm{~kg} / \mathrm{m}^{2}$ neither skinfold measurements nor BIA should be used. This statement is in line with the consensus recently published that BIA should be interpreted with caution in longitudinal follow-ups of body composition (BMI between 16 and $\left.34 \mathrm{~kg} / \mathrm{m}^{2}\right)^{(49)}$, in cases of malnourished individuals. Moreno et al. ${ }^{(30)}$ compared various BIA methods during refeeding against DXA in AN. They found that FFM derived from two commercially available BIA instruments (i.e. Tefal ${ }^{\circledR}$ and Xitron ${ }^{\circledR}$ ) was underestimated $v$. FFM derived from DXA measurements. Only one study has used multi-frequency BIA in order to differentiate between extracellular and intracellular water; Mika et al. ${ }^{(53)}$ found that multi-frequency BIA may be an appropriate tool for monitoring nutritional status during refeeding in AN patients; however, they did not use a 4-C model reference.

BIA in AN should be used with caution, but can still be clinically relevant in following up the relative changes in body compartments (rather than one punctual measurement) ${ }^{(32)}$ in patients with AN even if it is not yet validated against a 4-C model.

\section{Dual-energy X-ray absorptiometry}

Brief description of dual-energy X-ray absorptiometry. The DXA scanner has a scanning X-ray source, an X-ray detector that records absorption data at two energy levels, and an interface with a computer system for processing and analysing the absorption data. Body tissues absorb X-ray energy differently. Assumptions regarding the level of hydration, bone edge detection and body thickness are used to convert absorption data into mass values for bone, fat and lean tissue components. These measurements can be made for the entire body or specific body parts ${ }^{(17)}$. DXA is a valuable tool for body composition determination in research settings and in clinical settings it is mainly used for bone density assessment.

Applicability and limitation of dual-energy $X$-ray absorptiometry in anorexia nervosa. The limitations of DXA are related to the differences in the calibration for fat proportion between the different brands of DXA scanners and between models from the same manufacturer. Even small differences in calibration may lead to anomalous results in subjects with $\mathrm{AN}^{(19,54,55)}$. Moreover, DXA is associated with radiation exposure even if it is considered low; therefore it is not ideal to perform multiple measurements in children ${ }^{(17)}$. However, Blake et al. ${ }^{(56)}$ propose to use the infant whole body mode (only available on the A-model), and stress the fact that it is still important to keep radiation as small as possible, even though doses from child DXA examinations are low (for details about adjusting the DXA scan on infant modes, see Blake et $a l .{ }^{(56)}$ ). Recently, a study compared the body composition of premenopausal women, ranging from AN to obesity, with DXA and computed tomography (CT) scanning. They found that in AN, DXA is a useful method for assessing body composition. Measurements underestimated, compared with CT scanning, trunk FM by $10.7 \%$ and thigh fat by $3.8 \%$, with an overestimation of thigh muscle mass of $3.5 \%$. This might be due to the hydration status in AN, which can have a greater impact on DXA accuracy when assessing changes in body composition such as in treatment and follow-up ${ }^{(57,58)}$. Another study on AN female adolescents that compared DXA with skinfolds measurements found that, on an individual level, although body fat measurements from DXA and skinfolds were highly correlated, estimates of body fat were $9 \%$ higher or $6 \%$ lower when using SFT. This wide difference is not acceptable clinically in AN, as the limits of the BlandAltman agreements were wide. (The Bland-Altman plot is a statistical method that describes the agreement between two quantitative measurements; explained previously in Bland \& Altman ${ }^{(59)}$.) Even small differences in adiposity have great impact on physiological interpretations in patients with $\mathrm{AN}^{(60)}$. Some authors consider that DXA cannot be considered as a 'gold standard technology to assess fat mass' in $\mathrm{AN}^{(60)}$. Nevertheless, Helba \& Binkovitz ${ }^{(17)}$ concluded in their review article that DXA is the most accurate and reliable method of determining body composition in children with AN; it identifies accurately variations in FM and FFM in children and provides a better diagnostic and treatment follow-up tool of malnutrition in children with AN. In fact, DXA is accurate in measuring FM, but the value of FFM indicated by DXA does not indicate the level of hydration.

Since monitoring body composition in patients with AN is crucial for treatment and follow-up, BIA and DXA have been compared in several studies. In their study done on thirteen anorexic patients, Moreno et al. ${ }^{(30)}$ found that 
BIA instruments underestimated FFM in subjects with AN in comparison with DXA, but an adjustment in the software corrected this discrepancy. Additionally, DXA and BIA machines available on the market have different prediction equations and different physical characteristics, making their comparison with other techniques unreliable.

In their review, Pichard et al. ${ }^{(61)}$ explain that changes in body composition determine the patient's overall health status. Therefore, in patients with $\mathrm{AN}$, it is recommended to monitor systematically the variation levels between two measurements rather than the absolute values of FM and $\mathrm{FFM}^{(32)}$ because methods of measuring body composition are not yet validated against a 4-C model.

\section{Some other methods: computed tomography and underwater weighing}

Some other methods usually reserved for research purposes include CT and UWW.

Brief description of computed tomography. CT is an imaging technique that is particularly useful in studying the relative deposition of subcutaneous and intraabdominal fat ${ }^{(62)}$. However, its use is limited by the problems of availability (mostly used for diagnostic procedures other than nutritional assessment), radiation exposure and the high cost of the instrument.

Applicability and limitation of computed tomography in anorexia nervosa. In AN, many studies used this method in order to study regional fat distribution: Mayo-Smith et $a l .{ }^{(63)}$ used CT to measure subcutaneous and visceral adipose tissue in women with AN. They reported that the proportion of visceral adipose tissue to total adipose tissue in the CT image was significantly higher in patients with AN (0.4) compared with control subjects (0.14). Zamboni et al. ${ }^{(64)}$ replicated and extended the findings of Mayo-Smith et al. ${ }^{(63)}$ and they also concluded that patients with AN gain more in abdominal fat than other body regions.

Brief description of underwater weighing. UWW estimates FM starting from body density and applying mathematical equations. The body density is measured by submerging the body in water ${ }^{(65)}$. It is commonly used in order to validate other indirect methods of body composition measurements ${ }^{(66)}$.

Applicability and limitation of underwater weighing in anorexia nervosa. Probst et al. ${ }^{(21)}$ compared the skinfold method and the UWW method and tested their validity for evaluating body composition before and after weight gain in patients with AN with a BMI between 11.9 and $17 \cdot 5 \mathrm{~kg} / \mathrm{m}^{2}$. They examined the effects of weight changes on body composition and subcutaneous fat after a refeeding protocol. They found that body fat estimation by the SFT equation appeared to be as accurate as UWW with an acceptable error of percentage body fat between 2.5 and $3.8 \%$.

\section{Conclusion}

The success of the psychiatric treatment of patients with AN necessitates an improvement in their nutritional status. Unfortunately, body weight alone does not reflect the evolution of the different body compartments during refeeding in AN. Thus, it is beneficial that the treating team assesses the nutritional status of the patient according to methods that are accurate, reproducible and applicable clinically while keeping in mind the respective limitations of each method. The 'gold standard method' is the 4-C model which requires several hours for completion. Therefore, the choice of method for nutritional assessment in AN is mostly led by the techniques available and the objective of the assessment (routine $v$. research).

In clinical settings (i.e. follow-up of patients, punctual nutritional assessment, etc) a pragmatic recommendation is to maximise standardisation of the condition of measurements in order to get a homogeneous follow-up trend. When performed by trained personnel, weight, height and body impedance analysis are well adapted to the clinical setting for AN patients as well as MUAC and WHR. The latter is a very important tool in detecting the redistribution of the FM in AN patients. SFT might not be a sensitive tool for short-term weight-restored AN patients. DXA is a more expensive tool, but it also provides relevant information about bone mass status and can be used as one punctual assessment. A practical approach that is a reflective method for nutritional assessment and follow-up in AN and in clinical settings remains the use of at least two methods of assessment, for example, the BMI and BIA. It is recommended that the change between two periods is monitored rather than the isolated absolute values for any used method.

In research settings, more sophisticated methods such as UWW and CT can be considered if available in addition to DXA, BIA or SFT (for large sample population). As hydration and bone density cannot be considered stable in $\mathrm{AN}$, it is recommended that a combination of at least two methods is used that do not measure directly the same compartments such as DXA and body impedance analysis, SFT and BIA, etc. This will ensure that a high level of accuracy is obtained and it meets partially the concept of the 4-C model, which takes into account the different components of body composition and not only FM and FFM.

We still lack an accurate, unfailing and clinically applicable method of body composition measurement in AN patients. More studies are needed to further validate and standardise the different methods of body composition measurements in $\mathrm{AN}$.

\section{Acknowledgements}

We thank W. Mattar for help in the final editing of the article. L. M. receives grants from the Eiffel scholarship 
programme and C. P. receives grants from the public Foundation Nutrition 2000 Plus. N G. receives a grant from the 'Interface Contract' at INSERM-U669 (2004-2009). The funding source had no involvement in the writing of the review article and in the decision to submit the paper for publication.

L. M. managed the literature searches and wrote the majority of the present review article. J. C. M. assisted in critical proofreading of the whole manuscript and in correction, mainly of the parts on anthropometric measurements and the impact of AN on body composition. N. G. contributed to the meticulous correction of the whole article. C. P. supervised the whole process of writing, assisted in the writing of the part on bioelectrical impedance and undertook further corrections. All authors contributed to and have approved the final manuscript.

All authors of the present review article declare that they have no conflicts of interest or any other material benefit or profit.

\section{References}

1. Patton GC, Coffey C \& Sawyer SM (2003) The outcome of adolescent eating disorders: findings from the Victorian Adolescent Health Cohort Study. Eur Child Adolesc Psychiatry 12, Suppl. 1, I25-I29.

2. Steinhausen HC (2009) Outcome of eating disorders. Child Adolesc Psychiatr Clin N Am 18, 225-242.

3. Pollice C, Kaye WH, Greeno CG, et al. (1997) Relationship of depression, anxiety, and obsessionality to state of illness in anorexia nervosa. Int J Eat Disord 21, 367-376.

4. Meehan KG, Loeb KL, Roberto CA, et al. (2006) Mood change during weight restoration in patients with anorexia nervosa. Int J Eat Disord 39, 587-589.

5. Channon S \& DeSilva WP (1985) Psychological correlates of weight gain in patients with anorexia nervosa. $J$ Psychiatr Res 19, 267-271.

6. Wamboldt FS, Kaslow NJ, Swift WJ, et al. (1987) Short-term course of depressive symptoms in patients with eating disorders. Am J Psychiatry 144, 362-364.

7. Peterson MJ, Czerwinski SA \& Siervogel RM (2003) Development and validation of skinfold-thickness prediction equations with a 4-compartment model. Am J Clin Nutr 77, 1186-1191.

8. Heymsfield SB, Wang Z, Baumgartner RN, et al. (1997) Human body composition: advances in models and methods. Annu Rev Nutr 17, 527-558.

9. Withers RT, LaForgia J, Pillans RK, et al. (1998) Comparisons of two-, three-, and four-compartment models of body composition analysis in men and women. J Appl Physiol 85 , $238-245$.

10. Wells JC, Fuller NJ, Dewit O, et al. (1999) Four-component model of body composition in children: density and hydration of fat-free mass and comparison with simpler models. Am J Clin Nutr 69, 904-912.

11. Goran MI, Toth MJ \& Poehlman ET (1998) Assessment of research-based body composition techniques in healthy elderly men and women using the 4-compartment model as a criterion method. Int J Obes Relat Metab Disord 22, 135-142.

12. Kerruish KP, O'Connor J, Humphries IR, et al. (2002) Body composition in adolescents with anorexia nervosa. Am J Clin Nutr 75, 31-37.
13. Misra M, Aggarwal A, Miller KK, et al. (2004) Effects of anorexia nervosa on clinical, hematologic, biochemical, and bone density parameters in community-dwelling adolescent girls. Pediatrics 114, 1574-1583.

14. Mazess RB, Barden HS \& Hanson JA (1990) Body composition by dual-photon absorptiometry and dual-energy X-ray absorptiometry. Basic Life Sci 55, 427-432.

15. Schneider P, Biko J, Schlamp D, et al. (1998) Comparison of total and regional body composition in adolescent patients with anorexia nervosa and pair-matched controls. Eat Weight Disord 3, 179-187.

16. Miller KK, Wexler TL, Zha AM, et al. (2007) Androgen deficiency: association with increased anxiety and depression symptom severity in anorexia nervosa. J Clin Psychiatry 68, 959-965.

17. Helba M \& Binkovitz LA (2009) Pediatric body composition analysis with dual-energy X-ray absorptiometry. Pediatr Radiol 39, 647-656.

18. Misra M, Katzman DK, Cord J, et al. (2008) Bone metabolism in adolescent boys with anorexia nervosa. J Clin Endocrinol Metab 93, 3029-3036.

19. Shephard RJ (1991) Pathological disturbances of body composition. In Body Composition in Biological Anthropology, pp. 226-241. Cambridge: Cambridge University Press.

20. Iketani T, Kiriike N, Nagata T, et al. (1999) Altered body fat distribution after recovery of weight in patients with anorexia nervosa. Int J Eat Disord 26, 275-282.

21. Probst M, Goris M, Vandereycken W, et al. (2001) Body composition of anorexia nervosa patients assessed by underwater weighing and skinfold-thickness measurements before and after weight gain. Am J Clin Nutr 73, 190-197.

22. Russell DM, Prendergast PJ, Darby PL, et al. (1983) A comparison between muscle function and body composition in anorexia nervosa: the effect of refeeding. Am J Clin Nutr 38, 229-237.

23. Russell JD, Mira M, Allen BJ, et al. (1994) Protein repletion and treatment in anorexia nervosa. Am J Clin Nutr 59, 98-102.

24. Pichard C \& Kyle UG (1998) Body composition measurements during wasting diseases. Curr Opin Clin Nutr Metab Care 1, 357-361.

25. Melchior JC, Rigaud D, Rozen R, et al. (1989) Energy expenditure economy induced by decrease in lean body mass in anorexia nervosa. Eur J Clin Nutr 43, 793-799.

26. Gordon C, Chumlea W \& Roche A (1988) Stature, recumbent length, and weight. In Anthropometric Standardization Reference Manual, chapter 1, pp. 3-8 [TG Lohman, AR Roche and R Martorell, editors]. Champaign, IL: Human Kinetics Books.

27. Lee RD \& Neiman D (2003) Anthropometry. In Nutritional Assessment, 3rd ed., pp. 165-170. London: McGraw Hill.

28. Centers for Disease Control and Prevention (2010) Prevention CfDCa. http://www.cdc.gov/growthcharts/ clinical_charts.htm(accessed July 2010).

29. Golden NH, Jacobson MS, Sterling WM, et al. (2008) Treatment goal weight in adolescents with anorexia nervosa: use of BMI percentiles. Int J Eat Disord 41, 301-306.

30. Moreno MV, Djeddi DD \& Jaffrin MY (2008) Assessment of body composition in adolescent subjects with anorexia nervosa by bioimpedance. Med Eng Phys 30, 783-791.

31. Trocki O, Theodoros MT \& Shepherd RW (1998) Lack of sensitivity of weight targets compared with body cell mass for determining recovery from malnutrition in adolescents with anorexia nervosa. Int J Eat Disord 23, 169-176.

32. Kyle UG, Bosaeus I, De Lorenzo AD, et al. (2004) Bioelectrical impedance analysis - part I: review of principles and methods. Clin Nutr 23, 1226-1243. 
33. Trocki O \& Shepherd RW (2000) Change in body mass index does not predict change in body composition in adolescent girls with anorexia nervosa. J Am Diet Assoc 100, 457-460.

34. Cole TJ, Flegal KM, Nicholls D, et al. (2007) Body mass index cut offs to define thinness in children and adolescents: international survey. BMJ 335, 194.

35. Freedman DS, Wang J, Maynard LM, et al. (2005) Relation of BMI to fat and fat-free mass among children and adolescents. Int J Obes (Lond) 29, 1-8.

36. Golden NH, Jacobson MS, Schebendach J, et al. (1997) Resumption of menses in anorexia nervosa. Arch Pediatr Adolesc Med 151, 16-21.

37. Pollock M \& Jackson A (1984) Research progress in validation of clinical methods of assessing body composition. Med Sci Sports Exerc 16, 606-613.

38. Durnin JVGA \& Womersley J (1974) Body fat assessed from total body density and its estimation from skinfold thickness: measurements on 481 men and women aged from 16 to 72 years. Br J Nutr 32, 77-97.

39. Russell J, Allen B, Mira M, et al. (1994) Total body nitrogen as a predictor of clinical status in anorexia nervosa. Int J Eat Disord 15, 275-278.

40. Caregaro L, Favaro A, Santonastaso P, et al. (2001) Insulinlike growth factor 1 (IGF-1), a nutritional marker in patients with eating disorders. Clin Nutr 20, 251-257.

41. Misra M, Soyka LA, Miller KK, et al. (2003) Regional body composition in adolescents with anorexia nervosa and changes with weight recovery. Am J Clin Nutr 77, 1361-1367.

42. Collins S (1996) Using middle upper arm circumference to assess severe adult malnutrition during famine. JAMA 276, 391-395.

43. Martin AC, Pascoe EM \& Forbes DA (2009) Monitoring nutritional status accurately and reliably in adolescents with anorexia nervosa. J Paediatr Child Health 45, 53-57.

44. Steinkamp RC, Cohen NL, Siri WE, et al. (1965) Measures of body fat and related factors in normal adults. I. Introduction and methodology. J Chronic Dis 18, 1279-1291.

45. Forbes GB, Kreipe RE, Lipinski BA, et al. (1984) Body composition changes during recovery from anorexia nervosa: comparison of two dietary regimes. Am J Clin Nutr $\mathbf{4 0}$, 1137-1145.

46. Mayer L, Walsh BT, Pierson RN Jr, et al. (2005) Body fat redistribution after weight gain in women with anorexia nervosa. Am J Clin Nutr 81, 1286-1291.

47. Orphanidou CI, McCargar LJ, Birmingham CL, et al. (1997) Changes in body composition and fat distribution after short-term weight gain in patients with anorexia nervosa. Am J Clin Nutr 65, 1034-1041.

48. Maisonneuve N, Genton L, Karsegard VL, et al. (2004) Role of impedance measurement in nutritional screening (article in French). Rev Med Suisse Romande 124, 611-615.

49. Kyle UG, Bosaeus I, De Lorenzo AD, et al. (2004) Bioelectrical impedance analysis - part II: utilization in clinical practice. Clin Nutr 23, 1430-1453.

50. Bedogni G, Marra M, Bianchi L, et al. (2003) Comparison of bioelectrical impedance analysis and dual-energy X-ray absorptiometry for the assessment of appendicular body composition in anorexic women. Eur J Clin Nutr 57, 1068-1072.
51. Sun SS, Chumlea WC, Heymsfield SB, et al. (2003) Development of bioelectrical impedance analysis prediction equations for body composition with the use of a multicomponent model for use in epidemiologic surveys. Am J Clin Nutr 77, 331-340.

52. Piccoli A, Codognotto M, Di Pascoli L, et al. (2005) Body mass index and agreement between bioimpedance and anthropometry estimates of body compartments in anorexia nervosa. JPEN J Parenter Enteral Nutr 29, 148-156.

53. Mika C, Herpertz-Dahlmann B, Heer M, et al. (2004) Improvement of nutritional status as assessed by multifrequency BIA during 15 weeks of refeeding in adolescent girls with anorexia nervosa. J Nutr 134, 3026-3030.

54. Tothill P \& James Hannan W (2004) Dual-energy X-ray absorptiometry measurements of fat and lean masses in subjects with eating disorders. Int J Obes Relat Metab Disord 28, 912-919.

55. Genton L, Hans D, Kyle UG, et al. (2002) Dual-energy X-ray absorptiometry and body composition: differences between devices and comparison with reference methods. Nutrition 18, 66-70.

56. Blake GM, Naeem M \& Boutros M (2006) Comparison of effective dose to children and adults from dual X-ray absorptiometry examinations. Bone 38, 935-942.

57. Bredella MA, Ghomi RH, Thomas BJ, et al. (2010) Comparison of DXA and CT in the assessment of body composition in premenopausal women with obesity and anorexia nervosa Obesity (Silver Spring) (epublication ahead of print version 28 January 2010).

58. Hannan WJ, Cowen SJ, Freeman CP, et al. (1993) Evaluation of dual-energy X-ray absorptiometry for the assessment of body composition in anorexic females. Basic Life Sci $\mathbf{6 0}$, $169-172$.

59. Bland JM \& Altman DG (1986) Statistical methods for assessing agreement between two methods of clinical measurement. Lancet i, 307-310.

60. Haas VK, Kohn MR, Clarke SD, et al. (2009) Body composition changes in female adolescents with anorexia nervosa. Am J Clin Nutr 89, 1005-1010.

61. Pichard C, Genton L \& Jolliet P (2000) Measuring body composition: a landmark of quality control for nutritional support services. Curr Opin Clin Nutr Metab Care 3, 281-284.

62. Rogalla P, Meiri N, Hoksch B, et al. (1998) Low dose spiral computed tomography for measuring abdominal fat volume and distribution in a clinical setting. Eur J Clin Nutr 52, 597-602.

63. Mayo-Smith W, Hayes CW, Biller BM, et al. (1989) Body fat distribution measured with CT: correlations in healthy subjects, patients with anorexia nervosa, and patients with Cushing syndrome. Radiology 170, 515-518.

64. Zamboni M, Armellini F, Turcato E, et al. (1997) Body fat distribution before and after weight gain in anorexia nervosa. Int J Obes Relat Metab Disord 21, 33-36.

65. Probst M, Goris M, Vandereycken W, et al. (2008) Body composition in girls and young women with anorexia nervosa: comparison of different equations. Int $J$ Eat Disord $\mathbf{4 1}$, $180-183$.

66. Siri WE (1993) Body composition from fluid spaces and density: analysis of methods. 1961. Nutrition 9, 480-492. 